\title{
Projective system approach to the martingale characterization of the absence of arbitrage
}

\author{
Alejandro Balbás ${ }^{\mathrm{a}, *}$, Miguel Ángel Mirás ${ }^{\mathrm{b}}$, \\ María José Muñoz-Bouzo ${ }^{\mathrm{c}}$ \\ a Departmento de Economía de la Empresa, Universidad Carlos III de Madrid, \\ C/Madrid 126, 28903 Getafe, Madrid, Spain \\ ${ }^{\mathrm{b}}$ Departamento de Matemáticas, Facultad de Economía, Universidade de Vigo, \\ C/Lagoas Marcosende s/n, 36200 Vigo, Pontevedra, Spain \\ ${ }^{c}$ Departmento de Matemáticas Fundamentales, UNED, C/Senda del Rey 9, 28040 Madrid, Spain \\ Received 26 February 2001; received in revised form 24 April 2002; accepted 16 May 2002
}

\begin{abstract}
The equivalence between the absence of arbitrage and the existence of an equivalent martingale measure fails when an infinite number of trading dates is considered. By enlarging the set of states of nature and the probability measure through a projective system of perfect measure spaces, we characterize the absence of arbitrage when the time set is countable.
\end{abstract}

Keywords: Absence of arbitrage; Martingale measure; Asset pricing; Perfect measures; Projective systems

\section{Introduction}

For a frictionless securities market several authors, among others, Harrison and Kreps (1979), Taqqu and Willinger (1987), Dalang et al. (1990), Schachermayer (1992), Rogers (1994), Schachermayer (1994), Kabanov and Kramkov (1994), Delbaen and Schachermayer (1998), Jacod and Shiryaev (1998) and Pham and Touzi (1999) have proved different versions of the so-called Fundamental Theorem of Asset Pricing. In the case of a finite number of assets and a finite discrete time, this result simply states that the absence of arbitrage characterizes the existence of an equivalent martingale measure.

But things go wrong if one passes to infinite time (see Back and Pliska, 1991) or to infinitely many securities (see Schachermayer, 1992). In both situations, the characterization

\footnotetext{
* Corresponding author. Tel.: +34-91-624-9636; fax: +34-91-624-9608.

E-mail addresses: balbas@emp.uc3m.es (A. Balbás), mmiras@uvigo.es (M.Á. Mirás),mjmunoz@mat.uned.es (M.J. Muñoz-Bouzo).
} 
of an equivalent martingale measure for the price process of the assets needs notions, such as "no free lunch" or "no free lunch with bounded risk", generalizing the concept of "no arbitrage".

The purpose of this paper is to formulate the problem for infinite discrete time in a different mathematical setting, in order to obtain a theorem of asset pricing which may be phrased using only the classical notion of "no arbitrage". There are several reasons that inspire this goal. First, from an economic viewpoint, the concept of "arbitrage" is strictly weaker and far more intuitive than the concept of "free lunch". Second, the absence of arbitrage is, in general, easier to check in practical asset pricing models. Third, finding martingale probability measures in this new context allows us to analyze and price new securities if the arbitrage-free market is enlarged.

The construction of the martingale probability measure is addressed by drawing on projective systems of probability measures. This novel method is not only intuitive but also has clear economic interpretations. For instance, we can identify the set of states of nature with the set of admissible paths followed by the stochastic price process.

In securities market models with a finite number of assets, we show that the information needed to detect arbitrage opportunities can be limited to the filtrations formed by countably generated $\sigma$-algebras to which the price process of the stocks is adapted. Restricting our analysis to this particular class of filtrations is justified mathematically but it is also a financially sound assumption. In this setting, we construct a convergent projective system of perfect measure spaces whose limit extends the original space of states of nature and the probability measure. The absence of arbitrage is equivalent to the existence of a martingale measure on the projective limit space. Since, in general, the projective limit space strictly contains the initial space of states of nature, the original probability measure and the martingale measure are not equivalent. However, we show that the projections of both measures on every instant of time are equivalent. Therefore, there exist strictly positive Radon-Nikodym derivatives between the corresponding projections.

The paper is outlined as follows. Section 2 summarizes some basic notions and properties relative to projective systems of perfect measure spaces and introduces the securities market model. Since we only consider a finite number of securities, we prove in Section 3 that the absence of arbitrage can be characterized by restricting the information structure to a particular class of filtrations: those formed by countably generated $\sigma$-algebras. Section 4 develops the construction of a projective model for a market with a filtration formed by countably generated $\sigma$-algebras. Relying on a theorem on the convergence of projective systems of perfect measure spaces, we prove in Section 5 our main result, Theorem 5.4, that characterizes the absence of arbitrage by the existence of a projectively equivalent martingale measure. Finally, Section 6 concludes the paper.

\section{Preliminaries}

First, we recall the basic concepts and results on projective systems of measurable spaces, projective systems of measures and perfect measures. For further details see Musial (1980), Bourbaki (1969) or Bogachev (1998). 
Throughout this paper we use the standard set-theoretical terminology. Let $(\Omega, \Sigma)$ be an arbitrary measurable space. The $\sigma$-algebra $\Sigma$ is countably generated if there exists an at most countable family of subsets that generates $\Sigma$. We say that $\Sigma$ is separable if it is countably generated and contains all points of $\Omega$.

If $(X, \mathcal{F})$ is a measurable space, then for any $(\Sigma, \mathcal{F})$-measurable function $f: \Omega \rightarrow X$, the measure $\mu$ induces a measure on $\mathcal{F}$, called the image measure $f(\mu)$, given by $f(\mu)(A)=\mu\left(f^{-1}(A)\right)$ for all $A \in \mathcal{F}$.

Let $\leq$ be an ordering relation and $I$ a directed set. Consider a family of measurable spaces $\left(X_{i}, \Sigma_{i}\right)_{i \in I}$ and the $\left(\Sigma_{j}, \Sigma_{i}\right)$-measurable maps $\pi_{i j}: X_{j} \rightarrow X_{i} ; i, j \in I, i \leq j$. We say that $\left(X_{i}, \Sigma_{i}\right)_{i \in I}$ is a projective system of measurable spaces (or a measurable system) relative to maps $\pi_{i j}$, if $\pi_{i k}=\pi_{i j} \circ \pi_{j k}$ for all $i, j, k \in I, i \leq j \leq k$. The projective limit of $\left(X_{i}, \Sigma_{i}\right)_{i \in I}$ is the measurable space $(\bar{X}, \bar{\Sigma})$, where

$$
\bar{X}=\left\{\left(x_{i}\right)_{i \in I} \in \prod_{i \in I} X_{i}: x_{j}=\pi_{j k}\left(x_{k}\right), \quad \text { if } j, k \in I, j \leq k\right\},
$$

and $\bar{\Sigma}$ is the smallest $\sigma$-algebra of subsets of $\bar{X}$ relative to which the canonical projections $\pi_{i}: \bar{X} \rightarrow X_{i}, i \in I$, are $\left(\bar{\Sigma}, \Sigma_{i}\right)$-measurable. Note that $\bar{\Sigma}$ is the $\sigma$-algebra generated by $\left\{\cup \pi_{i}^{-1}\left(A_{i}\right): A_{i} \in \Sigma_{i}, i \in I\right\}$. Besides, $\pi_{i}=\pi_{i j} \circ \pi_{j}$ for all $i, j \in I, i \leq j$.

A family of measure spaces $\left(X_{i}, \Sigma_{i}, \mu_{i}\right)_{i \in I}$ is a projective system of measure spaces (shortly, a measure system) relative to maps $\pi_{i j}$, if $\left(X_{i}, \Sigma_{i}\right)_{i \in I}$ is a measurable system relative to maps $\pi_{i j}$ and $\pi_{i j}\left(\mu_{j}\right)=\mu_{i}$, if $i, j \in I, i \leq j$. The measure system is convergent if there is a measure $\bar{\mu}$ define on the projective limit $(\bar{X}, \bar{\Sigma})$ of $\left(X_{i}, \Sigma_{i}\right)_{i \in I}$ such that $\pi_{i}(\mu)=\mu_{i}$ for all $i \in I$. The measure space $(\bar{X}, \bar{\Sigma}, \bar{\mu})$ is called the projective limit of the measure system $\left(X_{i}, \Sigma_{i}, \mu_{i}\right)_{i \in I}$.

Let $(X, \mathcal{F})$ be a measurable space. A class $\mathcal{K}$ of subsets of $X$ is compact if for every sequence $\left\{K_{i}\right\}_{i=1}^{\infty} \subset \mathcal{K}$ such that $\bigcap_{i=1}^{s} K_{i} \not \equiv \emptyset$ for all $s \in \mathbb{N}$, one has $\bigcap_{i=1}^{\infty} K_{i} \neq \emptyset$. A measure $m$ on $(X, \mathcal{F})$ is compact (see Marczewski, 1953), if there exists a compact class $\mathcal{K}$ such that for every $F \in \mathcal{F}$ and $\varepsilon>0$ there exist $K \in \mathcal{K}$ and $E \in \mathcal{F}$ satisfying that $E \subset K \subset$ $F$ and $m(F \backslash E)<\varepsilon$. A measure $m$ on $(X, \mathcal{F})$ is perfect if for each measurable function $f$ : $X \rightarrow \mathbb{R}$ there exists $B$, a Borel set of $\mathbb{R}$, satisfying that $B \subset f(X)$ and $m\left(X \backslash f^{-1}(B)\right)=0$. Perfect measures are also called quasi-compact measures (Ryll-Nardzewski, 1953). There is a close connection between perfect and compact measures. Indeed, $m$ is perfect if and only if it is compact on every countably generated sub- $\sigma$-algebra of $\mathcal{F}$. Here are a number of elementary properties of perfect measures. If $m$ and $\ell$ are measures on $(X, \mathcal{F})$ and $m$ is absolutely continuous with respect to $\ell$, then $m$ is perfect if $\ell$ is such. The restriction of a perfect measure to any measurable subset and any sub- $\sigma$-algebra of measurable sets is again a perfect measure. Given any two measurable spaces $(X, \mathcal{F})$ and $(Y, \mathcal{G})$, if $m$ is a perfect measure on $(X, \mathcal{F})$ and $f: X \rightarrow Y$ is a $(\mathcal{F}, \mathcal{G})$-measurable function, then the image measure $f(m)$ on $(Y, \mathcal{G})$ is again a perfect measure.

The following result on the existence of a projective limit of a countable perfect measure system is adapted from Proposition 3.2 and Theorem 5.2 of Musial (1980).

Theorem 2.1. Suppose that $I=\mathbb{N}$. Every system $\left(X_{i}, \Sigma_{i}, \mu_{i}\right)_{i \in I}$ of perfectmeasure spaces relative to maps $\pi_{i j}$ such that $\Sigma_{i}$ is separable for all $i \in I$, converges to a unique separable perfect measure space $(\bar{X}, \bar{\Sigma}, \bar{\mu})$. 
Now, we describe our model of a frictionless financial market with a finite number of assets $n \in \mathbb{N}$ and a countable set of trading dates $T$. For convenience, let us take $T=$ $\mathbb{N} \cup\{0\}$. The information available to the agents at every time is described by a probability space $(\Omega, \Sigma, \mu)$ and an increasing family of sub- $\sigma$-algebras of $\Sigma,\left\{\Sigma_{t}\right\}_{t \in T}$, whose union generates $\Sigma$. Throughout the paper we assume that $\mu$ is a perfect probability measure. This is not a restrictive condition because the class of perfect measures is very large. Indeed (see Bogachev, 1998 for details), every compact measure is perfect and any tight measure (in particular, any Radon measure) is also perfect.

The prices of the risky stocks are given by a stochastic process $\left\{P\left(t,{ }_{-}\right)\right\}_{t \in T}$, with values in $\mathbb{R}^{n}$, adapted to the filtration $\left\{\Sigma_{t}\right\}_{t \in T}$. Naturally, for every $j=1, \ldots, n, \omega \in \Omega$ and $t \in T$, $P_{j}(t, \omega)$ is the price of asset $j$, if the true state of nature revealed in $t$ is $\omega$.

We suppose that the first security is a riskless bond. Without loss of generality (see Delbaen and Schachermayer, 1998) assume that the prices of the stocks have been discounted by the price of the bond, that is, take $P_{1}\left(t,{ }_{-}\right)=1$ for all $t \in T$.

Definition 2.2. The market is said to satisfy the absence of arbitrage if for every $t \in T$, $t \geq 1$, and every bounded $\Sigma_{t-1}$-measurable function $x: \Omega \rightarrow \mathbb{R}^{n}$

$$
\langle x(\omega), P(t, \omega)-P(t-1, \omega)\rangle=0, \quad \mu \text { almost surely, }
$$

whenever

$$
\langle x(\omega), P(t, \omega)-P(t-1, \omega)\rangle \geq 0, \quad \mu \text { almost surely, }
$$

where $\langle\cdot, \cdot\rangle$ denotes the inner product on $\mathbb{R}^{n}$.

The absence of arbitrage prevents the existence of zero cost portfolios with positive return. Any reasonable model of a financial market should satisfy this condition because, otherwise, some astute agent would take advantage of the arbitrage opportunities making riskless profits without investment.

\section{Countably generated filtrations}

The objective of this section is to find a characterization of the absence of arbitrage by means of the minimum amount of information needed to determine the prices of the stocks. Since we only consider a finite number of assets, we can restrict our attention to countably generated $\sigma$-algebras.

Definition 3.1. We say that an increasing family $\left\{\Sigma_{t}^{*}\right\}_{t \in T}$ is a countably generated subfiltration of $\left\{\Sigma_{t}\right\}_{t \in T}$ consistent with the price process $\left\{P\left(t,{ }_{-}\right)\right\}_{t \in T}$, if for every $t \in T, \Sigma_{t}^{*}$ is a countably generated sub- $\sigma$-algebra of $\Sigma_{t}$ such that $P\left(t,{ }_{-}\right)$is a $\Sigma_{t}^{*}$-measurable function.

Let us briefly describe one simple procedure to construct consistent countably generated sub-filtrations of $\left\{\Sigma_{t}\right\}_{t \in T}$. For every $t \in T$, take $\Sigma_{t}^{*}$ the smallest sub- $\sigma$-algebra of $\Sigma_{t}$ for which every function $P\left(s,{ }_{-}\right), s \leq t$, is $\Sigma_{t}^{*}$-measurable. Since the Borel measurable sets of $\mathbb{R}$ are countably generated and, for each $t \in T$, we deal with a finite number of 
functions, the family $\left\{\Sigma_{t}^{*}\right\}_{t \in T}$ is a countably generated sub-filtration of $\left\{\Sigma_{t}\right\}_{t \in T}$ consistent with the price process $\left\{P\left(t,{ }_{-}\right)\right\}_{t \in T}$.

Obviously, given $\left\{Q_{t}\right\}_{t \in T}$ a $\mathbb{R}^{d}$-valued stochastic process adapted to $\left\{\Sigma_{t}\right\}_{t \in T}$, a similar process can be applied to produce a consistent countably generated sub-filtration of $\left\{\Sigma_{t}\right\}_{t \in T}$ such that $\left\{Q_{t}\right\}_{t \in T}$ is also adapted to it. The notion of absence of arbitrage can be easily adjusted to fit a given consistent countably generated sub-filtration.

Definition 3.2. There is no arbitrage for $\left\{\Sigma_{t}^{*}\right\}_{t \in T}$, a consistent countably generated subfiltration of $\left\{\Sigma_{t}\right\}_{t \in T}$, if for every $t \in T, t \geq 1$, and every bounded $\Sigma_{t-1}^{*}$-measurable function $x: \Omega \rightarrow \mathbb{R}^{n}$, the same conditions of Definition 2.2 hold.

Now, we characterize the absence of arbitrage in the market in terms of countably generated sub-filtrations.

Proposition 3.3. The market satisfies the absence of arbitrage if and only if there is no arbitrage for every consistent countably generated sub-filtration of $\left\{\Sigma_{t}\right\}_{t \in T}$.

Proof. Trivially, the absence of arbitrage in the market implies that there is no arbitrage for any consistent countably generated sub-filtration. Conversely, suppose there exist $t_{0} \in T$, $t_{0} \geq 1$, and a bounded $\Sigma_{t_{0}-1}$-measurable function $x: \Omega \rightarrow \mathbb{R}^{n}$ such that $\left\langle x, P\left(t_{0}-1,_{-}\right)\right\rangle \leq$ 0 and $\left\langle x, P\left(t_{0},{ }_{-}\right)\right\rangle>0$. Let $\left\{\Sigma_{t}^{*}\right\}_{t \in T}$ be a countably generated sub-filtration such that the stochastic process $\left\{Q_{t}\right\}_{t \in T}$, where $Q_{t}=P\left(t,{ }_{-}\right)$if $t \not \equiv t_{0}-1$ and $Q_{t_{0}-1}=\left(P\left(t_{0}-1,{ }_{-}\right), x\right)$, is adapted to it. One can easily check that $x$ is an arbitrage opportunity for the new filtration, that is, $x$ is a $\Sigma_{t_{0}-1}^{*}$-measurable function that contradicts Definition 3.2.

This result allows us to turn our attention to the models of financial markets with countably generated filtrations.

\section{Discrete time projective models}

There are many instances of widely used $\sigma$-algebras that are countably generated; the Lebesgue measurable sets is just one of them. Besides, Proposition 3.3 asserts that this class of $\sigma$-algebras is enough to characterize the absence of arbitrage in the market. Therefore, in the remaining of the paper we assume that, for every $t \in T$, the $\sigma$-algebra $\Sigma_{t}$ has countable generators $\left\{G_{t}^{k}\right\}_{k \in \mathbb{N}}$.

Our goal in this section is to build a measure system whose projective limit is an extension of both the original space of states of nature and the probability measure.

The first phase consists in defining an adequate measurable system that enlarges the original space of states of nature. In order to do that, we define for every pair $(t, k) \in T \times \mathbb{N}$ a finite partition $\Delta_{t}^{k}$ of $\Omega$ as follows. Take $\Delta_{0}^{1}$ the partition of $\Omega$ induced by $G_{0}^{1}$. Next, by recurrence, if $t=0$ and $k>1$, let $\Delta_{0}^{k}$ be the partition of $\Omega$ generated by $G_{0}^{k}$ and the elements of $\Delta_{0}^{k-1}$. For $k=1$ and $t \in \mathbb{N}$, take $\Delta_{t}^{1}$ the partition of $\Omega$ induced by $G_{t}^{1}$ and the events that belong to $\Delta_{t-1}^{1}$. Finally, consider $\Delta_{t}^{k}$ the partition of $\Omega$ generated by $G_{t}^{k}$ and the elements of $\Delta_{t-1}^{k}$ and $\Delta_{t}^{k-1}$, whenever $t \in \mathbb{N}$ and $k>1$. 
Fix $t \in T$. First, we label the elements of $\Delta_{t}^{1}$ as $\Delta_{t}^{1}=\left\{A_{t, j}^{1}\right\}_{j=1}^{p_{1}^{t}}$. Next, we number the elements of $\Delta_{t}^{2}$ consecutively to the ones in $\Delta_{t}^{1}$, that is, $\Delta_{t}^{2}=\left\{A_{t, j}^{2}\right\}_{j=p_{1}^{t}+1}^{p_{2}^{t}}$. Proceeding like this, we obtain a new countable collection of generators of $\Sigma_{t}$, namely, $\left\{A_{t, j}^{k}\right\}_{j=1}^{\infty}$.

A couple of remarks are in place. Note that the index $j \in \mathbb{N}$ depends on the other indices $t$ and $k$, but, for the sake of simplicity, we do not explicitly specify that relationship. Observe, as well that, by construction, the new families of generators are intertwined, both in time and in the index $k$, having a double tree-like structure. Because, for our purposes, we only make use of the time structure, the details of the properties in the index $k$ are omitted. As for the time structure, to be precise, there exists a function $\varphi_{t}: \mathbb{N} \rightarrow \mathbb{N}$, onto, decreasing with $\varphi_{t}^{-1}(j)$ finite for every $j \in \mathbb{N}$, satisfying:

1. if $A_{t, j}^{k} \in \Delta_{t}^{k}$, then $A_{t-1, \varphi_{t}(j)}^{k} \in \Delta_{t-1}^{k}$ and $A_{t, j}^{k} \subset A_{t-1, \varphi_{t}(j)}^{k}$;

2. if $A_{t-1, h}^{k} \in \Delta_{t-1}^{k}$, then $A_{t, j}^{k} \in \Delta_{t}^{k}$ for every $j \in \varphi_{t}^{-1}(h)$. Moreover,

$$
A_{t-1, h}^{k}=\bigcup_{j \in \varphi_{t}^{-1}(h)} A_{t, j}^{k} .
$$

Let us go back to our construction of a measurable system that extends $\Omega$. Again, fix $t \in T$. We know that $\left(\Omega, \Sigma_{t}\right)$ is a measurable space with countable generators $\left\{A_{t, j}^{k}\right\}_{j=1}^{\infty}$. Let $F_{t}: \Omega \rightarrow[0,1]$ be the Marczewski function associated with $\left\{A_{t, j}^{k}\right\}_{j=1}^{\infty}$. Recall from Marczewski (1938) that $F_{t}$ is defined as

$$
F_{t}(\omega)=\sum_{j=1}^{\infty} \frac{2}{3^{j}} \chi\left(A_{t, j}^{k}\right)(\omega),
$$

where $\chi\left(A_{t, j}^{k}\right)$ is the characteristic function of $A_{t, j}^{k}$ and $F_{t}$ a $\Sigma_{t}$-measurable function. Denote $\Omega_{t}=F_{t}(\Omega) \subset[0,1]$. The Marczewski function identifies all the states of nature that belong to the same events of the countable family of generators. More explicitly, if $\alpha=F_{t}(\omega) \in \Omega_{t}$ and $J_{t}^{\omega}=\left\{j \in \mathbb{N}: \chi\left(A_{t, j}^{k}\right)(\omega) \not \equiv 0\right\}$, then $F_{t}^{-1}(\alpha)=\bigcap_{j \in J_{t}^{\omega}} A_{t, j}^{k}$. Take $\beta\left(\Omega_{t}\right)$ the Borel $\sigma$-algebra of $\Omega_{t}$. We define the map $H_{t}: \beta\left(\Omega_{t}\right) \rightarrow \Sigma_{t}$ by setting $H_{t}\left(B_{t}\right)=F_{t}^{-1}\left(B_{t}\right)$ for each $B_{t} \in \beta\left(\Omega_{t}\right)$. Once more from Marczewski (1938), we know that $\beta\left(\Omega_{t}\right)$ and $\Sigma_{t}$ are $\sigma$-isomorphic by $H_{t}$.

For each $t \in \mathbb{N}$ consider the map $\pi_{t-1, t}: \Omega_{t} \rightarrow \Omega_{t-1}$ given by $\pi_{t-1, t}(\alpha)=F_{t-1}(\omega)$ if $\alpha=F_{t}(\omega)$. Obviously, $\pi_{t-1, t}$ is well defined and is a $\left(\beta\left(\Omega_{t}\right), \beta\left(\Omega_{t-1}\right)\right)$-measurable function. In addition, $F_{t-1}=\pi_{t-1, t} \circ F_{t}$.

Hence, $\left(\Omega_{t}, \beta\left(\Omega_{t}\right)\right)_{t \in T}$ is a measurable system relative to maps $\pi_{t-1, t}$. Let $(\bar{\Omega}, \bar{\Sigma})$ be its projective limit and $\pi_{t}: \bar{\Omega} \rightarrow \Omega_{t}, t \in T$, the canonical projections.

Now, we replicate the filtration structure in the projective system. For every $t \in T$, let $\bar{\Sigma}_{t}$ be the $\sigma$-algebra on $\bar{\Omega}$ generated by $\left\{\pi_{t}^{-1}\left(B_{t, j}^{k}\right): j \in \mathbb{N}\right\}$ where $B_{t, j}^{k}=H_{t}^{-1}\left(A_{t, j}^{k}\right)$. Since, for every $t \in T, \beta\left(\Omega_{t}\right)$ and $\Sigma_{t}$ are $\sigma$-isomorphic by $H_{t},\left\{B_{t, j}^{k}\right\}_{j=1}^{\infty}$ is a countable collection of generators of $\beta\left(\Omega_{t}\right)$ and the family $\left\{\bar{\Sigma}_{t}\right\}_{t \in T}$ is a filtration on $\bar{\Omega}$.

Consider the map $L: \Omega \rightarrow \bar{\Omega}$ defined by $L(\omega)=\left(F_{t}(\omega)\right)_{t \in T}$. In simple terms, $L$ assigns to every state of nature $\omega$ the "path" or "trajectory" in $\bar{\Omega}$ formed by the points $\alpha_{t} \in[0,1]$ 
associated with the events $A_{t, j}^{k}$ to which $\omega$ belongs in every instant of time $t$. It is easy to check that $F_{t}=\pi_{t} \circ L$ for every $t \in T$. The map $L$ is a $(\Sigma, \bar{\Sigma})$-measurable function. Indeed, if $B_{t} \in \beta\left(\Omega_{t}\right), t \in T$, then $L^{-1}\left(\bigcup_{t=0}^{\infty} \pi_{t}^{-1}\left(B_{t}\right)\right) \in \Sigma$, because $\bigcup_{t=0}^{\infty} L^{-1}\left(\pi_{t}^{-1}\left(B_{t}\right)\right)=$ $\bigcup_{t=0}^{\infty}\left(\pi_{t} \circ L\right)^{-1}\left(B_{t}\right)=\bigcup_{t=0}^{\infty} F_{t}^{-1}\left(B_{t}\right) \in \Sigma_{t}$. Furthermore, $L$ is a $\left(\Sigma_{t}, \bar{\Sigma}_{t}\right)$-measurable function for all $t \in T$. It suffices to prove that for every $j \in \mathbb{N}$, the set $L^{-1}\left(\pi_{t}^{-1}\left(B_{t, j}^{k}\right)\right)=$ $A_{t, j}^{k}$ is a $\Sigma_{t}$-measurable set.

So far, we have enlarged the original space of states of nature through the projective limit of a measurable system. The next step is trying to extend the original probability measure. We accomplish that goal by constructing a convergent measure system. For every $t \in T$, consider the image measure $\mu_{t}=F_{t}(\mu)$. Since $\mu$ is perfect, $\mu_{t}$ is a perfect probability measure on $\beta\left(\Omega_{t}\right)$.

Proposition 4.1. The family $\left(\Omega_{t}, \beta\left(\Omega_{t}\right), \mu_{t}\right)_{t \in T}$ is a measure system of perfect probability spaces relative to maps $\pi_{t-1, t}$ and $\beta\left(\Omega_{t}\right)$ is separable for all $t \in T$.

Proof. First, observe that separability is straightforward. Therefore, we just have to prove that $\pi_{t-1, t}\left(\mu_{t}\right)=\mu_{t-1}$ for every $t \in \mathbb{N}$. Fix $t \in \mathbb{N}$ and $B_{t-1} \in \beta\left(\Omega_{t-1}\right)$. On one hand,

$$
\begin{aligned}
\pi_{t-1, t}\left(\mu_{t}\right)\left(B_{t-1}\right) & =\mu_{t}\left(\pi_{t-1, t}^{-1}\left(B_{t-1}\right)\right)=\mu\left(F_{t}^{-1}\left(\pi_{t-1, t}^{-1}\left(B_{t-1}\right)\right)\right) \\
& =\mu\left(\left(\pi_{t-1, t} \circ F_{t}\right)^{-1}\left(B_{t-1}\right)\right) .
\end{aligned}
$$

On the other hand, $\mu_{t-1}\left(B_{t-1}\right)=\mu\left(F_{t-1}^{-1}\left(B_{t-1}\right)\right)$. Since $F_{t-1}=\pi_{t-1, t} \circ F_{t}$, the last two expressions are equal.

According to Theorem 2.1, there exists a separable perfect measure $\bar{\mu}$ such that the projective system $\left(\Omega_{t}, \beta\left(\Omega_{t}\right), \mu_{t}\right)_{t \in T}$ converges to $(\bar{\Omega}, \underline{\Sigma}, \bar{\mu})$.

Proposition 4.2. The perfect probability measure $\bar{\mu}$ coincides with the image measure $L(\mu)$.

Proof. First of all, $L(\mu)$ is a perfect measure because $L$ is a $(\Sigma, \bar{\Sigma})$-measurable function. It remains to be shown that $\pi_{t}(L(\mu))=\mu_{t}$ for all $t \in T$. Take $t \in T$ and $B_{t} \in \beta\left(\Omega_{t}\right)$. Since $F_{t}=\pi_{t} \circ L$, the following chain of equalities is valid

$$
\begin{aligned}
\pi_{t}(L(\mu))\left(B_{t}\right) & =L(\mu)\left(\pi_{t}^{-1}\left(B_{t}\right)\right)=\mu\left(L^{-1}\left(\pi_{t}^{-1}\left(B_{t}\right)\right)\right)=\mu\left(\left(\pi_{t} \circ L\right)^{-1}\left(B_{t}\right)\right) \\
& =\mu\left(F_{t}^{-1}\left(B_{t}\right)\right)=\mu_{t}\left(B_{t}\right) .
\end{aligned}
$$

This concludes the proof.

Intuitively, $\bar{\mu}=L(\mu)$ is the extension of the probability measure $\mu$ to $\bar{\Omega}$. Finally, let $h: \Omega \rightarrow \mathbb{R}^{n}$ be a $\Sigma_{t}$-measurable function. Given $\alpha \in \Omega_{t}$, one can easily check that if $\alpha=F_{t}(\omega)=F_{t}\left(\omega^{\prime}\right)$ for some $\omega, \omega^{\prime} \in \Omega$ then $h(\omega)=h\left(\omega^{\prime}\right)$. Therefore, we can define the function $\bar{h}: \bar{\Omega} \rightarrow \mathbb{R}^{n}$ by setting, for every $\bar{\omega} \in \bar{\Omega}, \bar{h}(\bar{\omega})=h(\omega)$ where $\omega \in$ $F_{t}^{-1}\left(\pi_{t}(\bar{\omega})\right)$. Obviously, $\bar{h}$ is a $\bar{\Sigma}_{t}$-measurable function and extends the function $h$. In fact, 
$\bar{h}(L(\omega))=h(\omega)$, for all $\omega \in \Omega$. In particular, the stocks price process $\left\{P\left(t,{ }_{-}\right)\right\}_{t \in T}$ gives rise to a stochastic process $\left\{\bar{P}\left(t,{ }_{-}\right)\right\}_{t \in T}$ on $\bar{\Omega}$ adapted to the filtration $\left\{\bar{\Sigma}_{t}\right\}_{t \in T}$.

To sum up, we have enlarged our original securities market model to a new one, with the corresponding probability space $(\bar{\Omega}, \bar{\Sigma}, \bar{\mu})$, filtration $\left\{\bar{\Sigma}_{t}\right\}_{t \in T}$ and price process $\left\{\bar{P}\left(t,{ }_{-}\right)\right\}_{t \in T}$. This new description of the discrete time financial market will be called the projective model.

\section{Characterization of the absence of arbitrage in a projective model}

Assuming that every sub- $\sigma$-algebra of the filtration $\left\{\Sigma_{t}\right\}_{t \in T}$ has countable generators, we have been able to produce a projective model of the financial market. Our goal is to show that, in this new framework, the absence of arbitrage is equivalent to the existence of a martingale measure. We begin by translating the notion of absence of arbitrage to projective terms.

Proposition 5.1. The market satisfies the absence of arbitrage if and only iffor each $t \in T$, $t \geq 1$, and every bounded $\Sigma_{t-1}$-measurable function $x: \Omega \rightarrow \mathbb{R}^{n}$

$$
\langle\bar{x}(\bar{\omega}), \bar{P}(t, \bar{\omega})-\bar{P}(t-1, \bar{\omega})\rangle=0, \quad \bar{\mu} \text { almost surely, }
$$

whenever

$$
\langle\bar{x}(\bar{\omega}), \bar{P}(t, \bar{\omega})-\bar{P}(t-1, \bar{\omega})\rangle \geq 0, \quad \bar{\mu} \text { almost surely. }
$$

Now, we give a precise meaning to the idea of a "martingale measure" on the projective model. Since, as it will become clear in Example 5.6, one can not expect to find a martingale measure on $\bar{\Omega}$ equivalent to the extended probability measure $\bar{\mu}$, we introduce a weaker concept of equivalence.

Definition 5.2. We say that a measure $\bar{\lambda}$ on $\bar{\Omega}$ is projectively equivalent to $\bar{\mu}$, if $\lambda_{t}=\pi_{t}(\bar{\lambda})$ is equivalent to $\mu_{t}$ for all $t \in T$, i.e. if $\lambda_{t}$ and $\mu_{t}$ have the same null events.

Let us emphasize, and we refer the reader to Example 5.6, that two measures $\bar{\lambda}$ and $\bar{\mu}$ on $\bar{\Omega}$ can be projectively equivalent without being equivalent.

Definition 5.3. A risk-neutral projective probability measure (or projectively equivalent martingale measure) is a probability measure $\bar{\lambda}$ on $\bar{\Omega}$, projectively equivalent to $\bar{\mu}$, such that the stochastic process $\left\{\bar{P}\left(t,{ }_{-}\right)\right\}_{t \in T}$ is a martingale under $\bar{\lambda}$, i.e. for every $t \in T, t \geq 1$, $E_{\bar{\lambda}}\left[\bar{P}\left(t,{ }_{-}\right) \mid \bar{\Sigma}_{t-1}\right]=\bar{P}\left(t-1,{ }_{-}\right)$. Here, $E_{\bar{\lambda}}$ denotes the conditional expectation operator associated with $\bar{\lambda}$.

We can already state and prove the main result of this paper: the equivalence between the absence of arbitrage and the existence of a projectively equivalent martingale measure.

Theorem 5.4. The market satisfies the absence of arbitrage if and only if there exists a risk-neutral projective probability measure. 
Proof. First, we prove the necessity. Suppose that the market is arbitrage free. The Fundamental Theorem of Asset Pricing (Dalang et al., 1990; Schachermayer, 1992; Kabanov and Kramkov, 1994; Rogers, 1994; Jacod and Shiryaev, 1998) asserts that for any time interval of finite length, the absence of arbitrage implies the existence of an equivalent martingale measure. In particular, for every $t-1 \in T, t \geq 1$, there exists a probability measure $\theta_{t}$ on $\Sigma_{t}$, equivalent to $\mu_{t}$, such that $\left\{P\left(t-1,,_{-}\right), P\left(t,{ }_{-}\right)\right\}$is a martingale under $\theta_{t}$ and the filtration $\left\{\Sigma_{t-1}, \Sigma_{t}\right\}$, i.e. $E_{\theta_{t}}\left[P\left(t,{ }_{-}\right) \mid \Sigma_{t-1}\right]=P\left(t-1,{ }_{-}\right)$. Moreover, $\theta_{t}$ can be chosen such that the density $f_{t}=\left(\mathrm{d} \theta_{t} / \mathrm{d} \mu\right)>0$ is bounded (Schachermayer, 1992). Therefore, we can write

$$
P\left(t-1,{ }_{-}\right) E\left[f_{t} \mid \Sigma_{t-1}\right]=E\left[f_{t} P\left(t,{ }_{-}\right) \mid \Sigma_{t-1}\right] .
$$

The relation $1=E\left[f_{t} /\left(E\left[f_{t} \mid \Sigma_{t-1}\right]\right) \mid \Sigma_{t-1}\right]$ holds because $f_{t} /\left(E\left[f_{t} \mid \Sigma_{t-1}\right]\right) \in L^{1}\left(\Sigma_{t}\right)$. Consequently, for every $\Sigma_{t-1}$-measurable function $g: \Omega \rightarrow \mathbb{R}$, one has

$$
\int_{A_{t-1, h}^{k}} g \mathrm{~d} \mu=\int_{A_{t-1, h}^{k}} g \frac{f_{t}}{E\left[f_{t} \mid \Sigma_{t-1}\right]} \mathrm{d} \mu, \quad A_{t-1, h}^{k} \in \Sigma_{t-1} .
$$

So, in particular,

$$
P\left(t-1,_{-}\right)=E\left[P\left(t,,_{-}\right) \frac{f_{t}}{E\left[f_{t} \mid \Sigma_{t-1}\right]} \mid \Sigma_{t-1}\right] .
$$

The idea of this part of the proof is to build another convergent system $\left(\Omega_{t}, \beta\left(\Omega_{t}\right), \lambda_{t}\right)_{t \in T}$ of perfect measure spaces such that the projective limit measure $\bar{\lambda}$ on $\bar{\Sigma}$ has all the wanted properties. We divide the work in several steps.

Given $t \in T$, take the function $q_{t} \in L^{1}\left(\Sigma_{t}\right)$ defined by

$$
q_{t}= \begin{cases}1, & \text { if } t=0 \\ \frac{\prod_{j=1}^{t} f_{j}}{\prod_{j=1}^{t} E\left[f_{j} \mid \Sigma_{j-1}\right]}, & \text { if } t \geq 1\end{cases}
$$

Let $\lambda_{t}^{*}$ be the measure on $\Sigma_{t}$ whose Radon-Nikodym derivative with respect to $\mu$ is $q_{t}$, i.e. $q_{t}=\mathrm{d} \lambda_{t}^{*} / \mathrm{d} \mu$. Consider the image measure $\lambda_{t}=F_{t}\left(\lambda_{t}^{*}\right)$ on $\beta\left(\Omega_{t}\right)$. In other terms, given $B_{t} \in \beta\left(\Omega_{t}\right)$

$$
\lambda_{t}\left(B_{t}\right)=\lambda_{t}^{*}\left(F_{t}^{-1}\left(B_{t}\right)\right)=\int_{F_{t}^{-1}\left(B_{t}\right)} q_{t} \mathrm{~d} \mu .
$$

Since $q_{t}>0$, it is easy to derive that $\lambda_{t}$ and $\mu_{t}$ are equivalent measures for all $t \in T$. As a consequence, $\lambda_{t}$ is a perfect measure.

Step 1. The family $\left(\Omega_{t}, \beta\left(\Omega_{t}\right), \lambda_{t}\right)_{t \in T}$ is a system of perfect measure spaces relative to maps $\pi_{t-1, t}$. 
It suffices to prove that $\pi_{t-1, t}\left(\lambda_{t}\right)=\lambda_{t-1}$ for all $t \in \mathbb{N}$. Indeed, if $t \in \mathbb{N}$ and $B_{t-1} \in$ $\beta\left(\Omega_{t-1}\right)$, we have

$$
\begin{aligned}
\pi_{t-1, t}\left(\lambda_{t}\right)\left(B_{t-1}\right) & =\lambda_{t}\left(\pi_{t-1, t}^{-1}\left(B_{t-1}\right)\right)=\int_{F_{t}^{-1}\left(\pi_{t-1, t}^{-1}\left(B_{t-1}\right)\right)} q_{t} \mathrm{~d} \mu \\
& =\int_{\left(\pi_{t-1, t} \circ F_{t}\right)^{-1}\left(B_{t-1}\right)} q_{t} \mathrm{~d} \mu=\int_{F_{t-1}^{-1}\left(B_{t-1}\right)} q_{t} \mathrm{~d} \mu \\
& =\int_{F_{t-1}^{-1}\left(B_{t-1}\right)} \frac{f_{t}}{E\left[f_{t} \mid \Sigma_{t-1}\right]} q_{t-1} \mathrm{~d} \mu .
\end{aligned}
$$

Using relation (2), we can write

$$
\int_{F_{t-1}^{-1}\left(B_{t-1}\right)} \frac{f_{t}}{E\left[f_{t} \mid \Sigma_{t-1}\right]} q_{t-1} \mathrm{~d} \mu=\int_{F_{t-1}^{-1}\left(B_{t-1}\right)} q_{t-1} \mathrm{~d} \mu=\lambda_{t-1}\left(B_{t-1}\right) .
$$

Combining (4) and (5) yields the wanted equality.

Step 2. For every $t \in \mathbb{N}, \lambda_{t}^{*}$ is an extension of $\lambda_{t-1}^{*}$.

We will show that $\lambda_{t}^{*}$ and $\lambda_{t-1}^{*}$ take the same values on the generators $A_{t-1, h}^{k}$ of $\Sigma_{t-1}$. We know that $A_{t-1, h}^{k}=\bigcup_{j \in \varphi_{t}^{-1}(h)} A_{t, j}^{k}$, with $A_{t, j}^{k} \in \Sigma_{t}$ for all $j \in \varphi_{t}^{-1}(h)$. We have

$$
\lambda_{t-1}^{*}\left(A_{t-1, h}^{k}\right)=\int_{A_{t-1, h}^{k}} q_{t-1} \mathrm{~d} \mu=\int_{\bigcup_{j \in \varphi_{k}^{-1}(h)} A_{t, j}^{k}} q_{t-1} \mathrm{~d} \mu=\sum_{j \in \varphi_{k}^{-1}(h)} \int_{A_{t, j}^{k}} q_{t-1} \mathrm{~d} \mu .
$$

By relation (2), we can continue writing

$$
\begin{aligned}
\sum_{j \in \varphi_{k}^{-1}(h)} \int_{A_{t, j}^{k}} q_{t-1} \mathrm{~d} \mu & =\sum_{j \in \varphi_{k}^{-1}(h)} \int_{A_{t, j}^{k}} q_{t-1} \frac{f_{k}}{E\left[f_{k} \mid \Sigma_{t-1}\right]} \mathrm{d} \mu \\
& =\int_{\bigcup_{j \in \varphi_{k}^{-1}(h)} A_{t, j}^{k}} q_{t} \mathrm{~d} \mu=\lambda_{t}^{*}\left(A_{t-1, h}^{k}\right) .
\end{aligned}
$$

Step 3. For every $s \in \mathbb{N}$, the finite price process $\left\{P\left(t,{ }_{-}\right)\right\}_{t=0}^{s}$ is a martingale with respect to the measure $\lambda_{s}^{*}$ and the filtration $\left\{\Sigma_{t}\right\}_{t=0}^{s}$.

Take $t \in\{1, \ldots, s\}$. Multiplying (3) by $q_{t-1}$ yields

$$
P\left(t-1,{ }_{-}\right) q_{t-1}=E\left[P\left(t,{ }_{-}\right) q_{t} \mid \Sigma_{t-1}\right] .
$$

Therefore,

$$
\int_{A_{t-1, h}^{k}} P\left(t-1,,_{-}\right) \mathrm{d} \lambda_{t-1}^{*}=\int_{A_{t-1, h}^{k}} P\left(t,,_{-}\right) \mathrm{d} \lambda_{t}^{*}, \quad A_{t-1, h}^{k} \in \Sigma_{t-1} .
$$


By Step 2,

$$
\int_{A_{t-1, h}^{k}} P\left(t-1,,_{-}\right) \mathrm{d} \lambda_{t-1}^{*}=\int_{A_{t-1, h}^{k}} P\left(t-1,{ }_{-}\right) \mathrm{d} \lambda_{t}^{*}, \quad A_{t-1, h}^{k} \in \Sigma_{t-1} .
$$

Finally, equalities (6) and (7) produce $P\left(t-1,{ }_{-}\right)=E_{\lambda_{t}^{*}}\left[P\left(t,{ }_{-}\right) \mid \Sigma_{t-1}\right]$. Now, the result is an immediate consequence of the fact that $\lambda_{s}^{*}$ is an extension of the measures $\lambda_{t}^{*}, t \in$ $\{0, \ldots, s-1\}$.

Step 4. The measure system $\left(\Omega_{t}, \beta\left(\Omega_{t}\right), \lambda_{t}\right)_{t \in T}$ converges to a separable perfect measure space $(\bar{\Omega}, \bar{\Sigma}, \bar{\lambda})$ and $\bar{\lambda}$ is projectively equivalent to $\bar{\mu}$.

By Step 2 and the separability of $\beta\left(\Omega_{t}\right)$, Theorem 2.1 guarantees the existence of a separable perfect measure $\bar{\lambda}$ such that $\left(\Omega_{t}, \beta\left(\Omega_{t}\right), \lambda_{t}\right)_{t \in T}$ converges to $(\bar{\Omega}, \bar{\Sigma}, \bar{\lambda})$. As $\lambda_{t}$ and $\mu_{t}$ are equivalent for all $t \in \mathbb{N}$, the measures $\bar{\lambda}$ and $\bar{\mu}$ are projectively equivalent.

Step 5. The process $\left\{\bar{P}\left(t,{ }_{-}\right)\right\}_{t \in T}$ is a martingale with respect to $\bar{\lambda}$ and the filtration $\left\{\bar{\Sigma}_{t}\right\}_{t \in T}$.

We have to prove that, for all $h \in \mathbb{N}$

$$
\int_{\pi_{t-1}^{-1}\left(B_{t-1, h}^{k}\right)} \bar{P}\left(t-1,,_{-}\right) \mathrm{d} \bar{\lambda}=\int_{\pi_{t-1}^{-1}\left(B_{t-1, h}^{k}\right)} \bar{P}\left(t,{ }_{-}\right) \mathrm{d} \bar{\lambda} .
$$

Observe that

$$
\int_{\pi_{t-1}^{-1}\left(B_{t-1, h}^{k}\right)} \bar{P}\left(t-1,{ }_{-}\right) \mathrm{d} \bar{\lambda}=\int_{A_{t-1, h}^{k}} P\left(t-1,{ }_{-}\right) \mathrm{d} \lambda_{t-1}^{*},
$$

and

$$
\int_{\pi_{t-1}^{-1}\left(B_{t-1, h}^{k}\right)} \bar{P}\left(t,{ }_{-}\right) \mathrm{d} \bar{\lambda}=\int_{A_{t-1, h}^{k}} P\left(t-1,{ }_{-}\right) \mathrm{d} \lambda_{t}^{*} .
$$

Now, the result follows directly from (7). In summary, $\bar{\lambda}$ is a risk-neutral projective probability measure and the necessity part is completed.

We turn now to the sufficiency. Let $\bar{\lambda}$ be a projectively equivalent martingale measure. Then, for every $\bar{\Sigma}_{t-1}$-measurable bounded function $\bar{h}: \bar{\Omega} \rightarrow \mathbb{R}^{n}$

$$
E_{\bar{\lambda}}\left[\left\langle\bar{h}, \bar{P}\left(t,{ }_{-}\right)-\bar{P}\left(t-1,{ }_{-}\right)\right\rangle\right]=0 .
$$

If, in addition, $\langle\bar{h}(\bar{\omega}), \bar{P}(t, \bar{\omega})-\bar{P}(t-1, \bar{\omega})\rangle \geq 0, \bar{\mu}$ almost surely, then we have $\langle\bar{h}(\bar{\omega})$, $\bar{P}(t, \bar{\omega})-\bar{P}(t-1, \bar{\omega})\rangle \geq 0, \bar{\lambda}$ almost surely, because $\bar{\mu}$ and $\bar{\lambda}$ are projectively equivalent. Then, from (9) and Proposition 5.1 we conclude that the absence of arbitrage holds in the market.

Example 5.5. As a particular case, we want to indicate how our construction applies to the practical case of a model in which every $\Sigma_{t}, t \in T$, is generated by a countable partition $\left(A_{t}^{j}\right)_{j=1}^{\infty}$ of $\Omega$ formed by events of positive probability. The partitions can be modified to have a tree-like structure on time. Then, it can be proved that every $\Omega_{t}$ is a sequence of 
points of $[0,1]$. Furthermore, $\Omega_{t}$ can be put in a one-to-one correspondence with the set of events $\left\{A_{t}^{j}: j \in \mathbb{N}\right\}$. So, $\bar{\Omega}$ can be view as the space of admissible trajectories following the branches of the tree. Since, for every $t \in T$, the family $\left(A_{t}^{j}\right)_{j=1}^{\infty}$ is a partition of $\Omega$, given $\omega \in \Omega$ there exists a unique $t(\omega) \in \mathbb{N}$ such that $\omega \in A_{t}^{t(\omega)}$. Therefore, the map $L$ identifies every state of nature $\omega \in \Omega$ with the "path" or "trajectory" in $\bar{\Omega}$ formed by the events $A_{t}^{t(\omega)}$ to which $\omega$ belongs in each instant of time $t \in T$.

Example 5.6 (Back and Pliska). Let us examine, under our method, the example of Back and Pliska (1991). Imagine the random experiment of rolling a fair die until the first number different from 6 comes out. Denote by $\omega \in \mathbb{N}$ the number of the roll when this occurs. Clearly, the probability of every event $\omega \in \mathbb{N}$ is $\mu(\omega)=(5 / 6)(1 / 6)^{\omega-1}$. Suppose that only two securities can be sold and bought every time $t \in \mathbb{N}$ that we roll the die. The first one is the riskless bond. The price process of the second security is

$$
P_{2}(t, \omega)= \begin{cases}1, & \text { if } t=0 \\ \left(\frac{1}{2}\right)^{t}, & \text { if } 0<t<\omega \\ \left(\omega^{2}+2 \omega+2\right)\left(\frac{1}{2}\right)^{\omega}, & \text { if } t \geq \omega\end{cases}
$$

There are no arbitrage opportunities in this market but no measure on $\mathbb{N}$ is an equivalent martingale measure to $\mu$.

It is easy to check that, for this example, $\bar{\Omega}=\mathbb{N} \cup\{\infty\}$ and $\bar{\Sigma}$ coincides with the Borel $\sigma$-algebra associated with the Alexandroff compactification of $\mathbb{N}$. The projective model just adds one more event corresponding to the point of infinity: "number 6 comes out in all the rolls". Obviously, the point of infinity is a null event, i.e. $\bar{\mu}(\infty)=0$. Since the market is arbitrage free, there must be a projectively equivalent martingale measure. Following the constructive procedure of Theorem 5.4, one finds that the measure $\bar{\lambda}(\omega)=1 /(2 \omega(\omega+1))$, $\bar{\lambda}(\infty)=1 / 2$, is a risk-neutral projective probability measure.

Observe that $\bar{\lambda}$ assigns positive probability to the $\bar{\mu}$-null event $\infty$ and, consequently, $\bar{\mu}$ and $\bar{\lambda}$ are not equivalent measures. However, as pointed out by Theorem 5.4, $\bar{\mu}$ and $\bar{\lambda}$ are projectively equivalent.

\section{Conclusions}

For an infinite number of trading dates the characterization of the absence of arbitrage by the existence of equivalent martingale measures presents some difficulties, and the price process of the assets needs notions such as "no free lunch" or "no free lunch with bounded risk", generalizing the concept of "no arbitrage".

This paper has formulated the problem for infinite discrete time in a different mathematical setting, and it has obtained a theorem of asset pricing which may be phrased using only the classical notion of "no arbitrage".

The martingale measure is built as a projective limit of perfect measures and extends the initial probability. Both the martingale measure and the initial probability measure generate equivalent projections. 


\section{Acknowledgements}

This research has been supported by grant BEC2000-1388-C04 from the Spanish Ministry of Science and Technology. We also want to thank an anonymous referee for his/her suggestions and comments that have greatly help us improving this paper.

\section{References}

Back, K., Pliska, S.R., 1991. On the fundamental theorem of asset pricing with an infinite state space. Journal of Mathematical Economics 20, 1-18.

Bogachev, V., 1998. Measures on topological spaces. Journal of Mathematical Sciences 91 (4), 3033-3156.

Bourbaki, N., 1969. Intégration. In: Eléments de Mathématique. Diffusion C.C.L.S., Paris (Chapitre IX).

Dalang, R.C., Morton, A., Willinger, W., 1990. Equivalent martingale measures and no arbitrage in stochastic securities market models. Stochastics and Stochastic Reports 29, 185-201.

Delbaen, F., Schachermayer, W., 1998. The fundamental theorem of asset pricing for unbounded stochastic processes. Mathematische Annalen 312 (2), 215-250.

Harrison, M., Kreps, D.M., 1979. Martingale and arbitrage in multiperiod security markets. Journal of Economic Theory 20, 381-408.

Jacod, J., Shiryaev, A., 1998. Local martingales and the fundamental asset pricing theorems in the discrete time case. Finance and Stochastics 2 (3), 259-273.

Kabanov, Y., Kramkov, D., 1994. No arbitrage and equivalent martingale measures: an elementary proof of the Harrison-Pliska theorem. Theory of Probability and its Applications 39, 523-526.

Marczewski, E., 1938. The characteristic function of a sequence of sets and some of its applications. Fundamenta Mathematicae 31, 207-229.

Marczewski, E., 1953. On compact measures. Fundamenta Mathematicae 40, 113-124.

Musial, K., 1980. Projective limits of perfect measure spaces. Fundamenta Mathematicae 40, 163-189.

Pham, H., Touzi, N., 1999. The fundamental theorem of asset pricing with cone constraints. Journal of Mathematical Economics 31, 265-279.

Rogers, L., 1994. Equivalent martingale measures and no arbitrage. Stochastics and Stochastic Reports 51, 41-49.

Ryll-Nardzewski, C., 1953. On quasi-compact measures. Fundamenta Mathematicae 40, 125-130.

Schachermayer, W., 1992. A Hilbert space proof of the fundamental theorem of asset pricing in finite discrete time. Insurance: Mathematics and Economics 11 (4), 249-257.

Schachermayer, W., 1994. Martingale measures for discrete time processes with infinite horizon. Mathematical Finance 4 (1), 25-55.

Taqqu, M.S., Willinger, W., 1987. The analysis of finite security markets using martingales. Advances in Applied Probability 19, 1-25. 